\title{
АНАЛИЗ ЭФФЕКТИВНОСТИ ПРОМЕЖУТОЧНОГО ПЕРЕГРЕВА ПАРА В КОТЛЕ-УТИЛИЗАТОРЕ ГТ-НАДСТРОЙКИ ТЕПЛОФИКАЦИОННОГО ЭНЕРГОБЛОКА
}

\author{
Минор Александр Александрович1, \\ aam45@tpu.ru \\ Ромашова Ольга Юрьевна 1 , \\ roma@tpu.ru \\ 1 Национальный исследовательский Томский политехнический университет,
Россия, 634050, г. Томск, пр. Ленина, 30.
}

\begin{abstract}
Актуальность. Модернизация существующего теплоэнергетического оборудования является одним из приоритетных направлений развития энергетики. Внедрение газотурбинной установки в тепловую схему паросилового энергоблока $c$ сохранением инфраструктуры станции может быть менее затратным вариантом по сравнению с сооружением новых энергоблоков парогазовых установок. Одним из малозатратных вариантов интеграции газотурбинных установок в тепловую схему паротурбинных энергоблоков является использование тепловой мощности котла-утилизатора для промежуточного перегрева отработавшего в паровой турбине рабочего тела с утилизацией оставщейся теплоты для нагрева питательной воды и основного конденсата. По сравнению с наиболее распространенными схемами с вытеснением регенерации в котелутилизатор предложенный альтернативный вариант позволяет повысить тепловую экономичность паротурбинного контура. Такая схема уступает в тепловой экономичности схеме с параллельной генерацией пара в котле-утилизаторе, однако не требует испарительного контура, что упрощает проектирование котла-утилизатора. Проведенный анализ энергетических характеристик газотурбинных установок показал необходимость исследования схемы и параметров промежуточного перегрева для теплосикационных энергоблоков с учетом режимов работы паровой турбины.

Объект: паротурбинный энергоблок T-165/210-130 с газотурбинной надстройкой (ГТ-надстройкой) для промежуточного перегрева отработавшего в паровой турбине рабочего тела с утилизацией оставшейся теплоты для нагрева питательной воды и основного конденсата.

Цель: выбор схемы и расчет оптимальных параметров промежуточного перегрева применительно к паротурбинному энергоблоку T-165/210-130 с ГТ-надстройкой, анализ его основных показателей при работе по тепловому графиику при изменении температуры промежуточного перегрева в котле-утилизаторе

Методы: системный анализ и математическое моделирование.

Результаты. Разработана математическая модель и программа расчета предложенной схемы. Выполнен анализ особенностей, связанных с организацией промежуточного перегрева в котле-утилизаторе ГТ-надстройки. Показано, что эффрективность работы теплофикационного энергоблока с промежуточным перегревом может быть повышена путем организации промежуточного перегрева в котле-утилизаторе ГТ-надстройки. Определено, что в этом случае наивысшая экономичность паротурбинного энергоблока с ГТ-надстройкой при работе в расчетном теплофикационном режиме достигается при температуре промежуточного перегрева ниже номинального значения.
\end{abstract}

\section{Ключевые слова:}

Промежуточный перегрев, парогазовая установка, газотурбинная надстройка, котел-утилизатор, теплофикационньй режим, удельный расход топлива, коэффициент использования теплоты топлива, расход пара, электрическая мощность, тепловая нагрузка.

\section{Введение}

Одним из приоритетных направлений развития энергетики является модернизация существующего теплоэнергетического оборудования. На современном этапе развития энергетики России отдается предпочтение внедрению газотурбинных технологий, что обусловлено значительными запасами природного газа. Парогазовые установки (ПГУ) утилизационного типа считаются наиболее совершенными установками для производства электроэнергии (ЭЭ) [1]. Наряду с наиболее эффективным, но и более затратным, вариантом сооружения современных энергоблоков ПГУ, имеет место переход на газотурбинные технологии с сохранением инфраструктуры существующей станции, что позволяет интегрировать газотурбинную установку (ГТУ) в тепловую схему паросилового блока (в том числе и на пылеугольных электростанциях, где природный газ используется как второе топливо) с меньшими инвестиционными расходами $[2,3]$.
Дополнительный эффект ГТ-надстройки могут дать в сочетании с переводом конденсационных энергоблоков в теплофикационный режим при необходимости вытеснения неэкономичных районных котельных. При этом наряду с увеличением отпуска теплоты внешнему потребителю в отопительный период увеличивается электрическая мощность станции. В результате снижается расход топлива по сравнению с раздельной выработкой теплоты и электроэнергии, а также уменьшаются удельные значения тепловых и вредных выбросов в атмосферу. Такая модернизация ведет к сокращению затрат на газо- и угледобычу.

Среди малозатратных вариантов интеграции газовой турбины (ГТ) в тепловую схему паротурбинных энергоблоков наибольшее распространение получили схемы с подогревом питательной воды (ПВ) и основного конденсата (ОК) в котле-утилизаторе (КУ) [4-6].

Недостатком такого варианта совершенствования ТЭС является существенное ухудшение показателей 
тепловой экономичности паротурбинной установки (ПТУ): растет доля потерь теплоты в конденсаторе, снижается расход острого пара по сравнению с номинальным из-за ограничения пропуска пара через последние ступени паровой турбины (ПТ) с соответствующим падением электрической мощности.

Альтернативным способом вытеснению регенерации на блочных ТЭС может служить вариант использования тепловой мощности КУ для промежуточного перегрева отработавшего в ПТ рабочего тела с утилизацией оставшейся теплоты для нагрева ПВ и ОК. Преимущества в экономичности такого решения для конденсационных энергоблоков по сравнению с вытеснением регенерации показаны в работе [7]. Предложенный вариант уступает в тепловой экономичности схеме с параллельной генерацией пара в КУ, однако не требует испарительного контура, что упрощает проектирование котла-утилизатора. Это преимущество объединяет его со схемой с вытеснением регенерации, однако, в отличие от последней, рассмотренный вариант позволяет повысить тепловую экономичность [8, 9] паротурбинного контура.

Анализ энергетических характеристик ГТУ показал необходимость исследования схемы и параметров промежуточного перегрева (ПП) для теплофикационных блоков с учетом изменения режимов работы ПТ в зависимости от температуры наружного воздуха $[5,10]$. При этом следует учитывать, что для теплофикационных энергоблоков вопрос об использовании ПП является дискуссионным [11], так как в конденсационном и теплофикационном режимах он имеет разную степень эффективности.

Известно, что для всех конденсационных турбин с начальным давлением пара 12,8 и 23,5 МПа принят промежуточный перегрев, который приводит к повышению тепловой экономичности и снижению влажности в последних ступенях. Для теплофикационных турбин эффективность ПП меньше, чем для конденсационных. Кроме того, на характерном для теплофикационных турбин режиме по тепловому графику с минимально необходимым для охлаждения последних ступеней турбины расходом пара в конденсатор $G_{\mathrm{K}}{ }^{\mathrm{min}}$ температура пара на выходе из турбины при ПП возрастает, что требует или увеличения $G_{\mathrm{K}}^{\min }$, или применения специальной системы охлаждения, как это выполнено в турбине Т-250/300-240. При этом положительный эффект от увеличения удельной выработки ЭЭ на тепловом потреблении за счет ПП не всегда превышает потерю экономичности из-за роста потери теплоты в конденсаторе при работе с закрытой диафрагмой части низкого давления (ЧНД).

По этой причине теплофикационные турбины Уральского турбинного завода с давлением свежего пара 12,8 МПа типа Т-110-130, Т-175-130 и другие, в отличие от конденсационных энергоблоков на это давление (К-160-130, К-210-130), выполняются без ПП.
В связи с вышесказанным актуальным становится исследование показателей работы теплофикационного энергоблока при работе по тепловому графику при изменении температуры промежуточного перегрева, требующей изменения минимально необходимого для охлаждения последних ступеней турбины расхода пара в конденсатор.

\section{Постановка задачи}

Работа посвящена выбору схемы и расчету оптимальных параметров [12-15] промежуточного перегрева пара применительно к паротурбинному энергоблоку Т-165/210-130 с ГТ-надстройкой (в дальнейшем упоминается в тексте как ПГУ). Турбоагрегат T-165/210-130 принят в качестве прототипа, его схема и параметры определены на основе материалов по переводу конденсационной турбины К-210-130 в теплофикационный режим с регулируемым отопительным отбором традиционным способом на одной из станций Уральского федерального округа. Такая схема реконструкции подразумевает подогрев сетевой воды по одноступенчатой схеме в основном сетевом подогревателе, на который подается пар из регулируемого отбора. Регулируемый отбор пара осуществляется из камеры за цилиндром среднего давления (ЦСД), где имеется возможность отвода его необходимого количества. Отвод пара производится из переходного патрубка выхлопной части ЦСД. Изменение расхода пара в регулируемый отбор осуществляется главным образом изменением расхода пара в цилиндр низкого давления (ЦНД). Для этого перед ЦНД устанавливается клапан типа поворотной заслонки [16].

На рис. 1 представлена принципиальная тепловая схема разрабатываемой ПГУ. Надстроенная часть включает в себя газотурбинную установку, котелутилизатор, состоящий из трех частей - пароперегреватель (ПЕ) для перегрева отработавшего в цилиндре высокого давления (ЦВД) пара, газоводяные подогреватели высокого (ГВП ВД) и низкого (ГВП НД) давления для нагрева питательной воды и основного конденсата.

В основу расчетов положена зависимость минимально необходимого расхода пара $G_{\mathrm{K}}^{\text {min }}$ для охлаждения последних ступеней турбины К-210-130 от энтальпии на входе в ЧНД, представленная графически на рис. 2. График получен с использованием аналитических зависимостей из [17] и практических результатов эксплуатационных испытаний турбины Т-178/210-130 [18]. Расход охлаждающего пара в ЧНД при значениях давления в конденсаторе $0,0035 \ldots 0,005$ МПа и номинальной температуре промежуточного перегрева составляет 140-207 т/ч, что значительно выше $G_{\text {к }}^{\text {min }}$ для теплофикационных турбин без промежуточного перегрева с такими же начальными параметрами пара. Увеличение $G_{\text {к }}{ }^{\text {min }}$ при заданном расходе острого пара означает существенное ограничение отпуска теплоты внешнему потребителю при переводе конденсационной турбины в теплофикационный режим. 


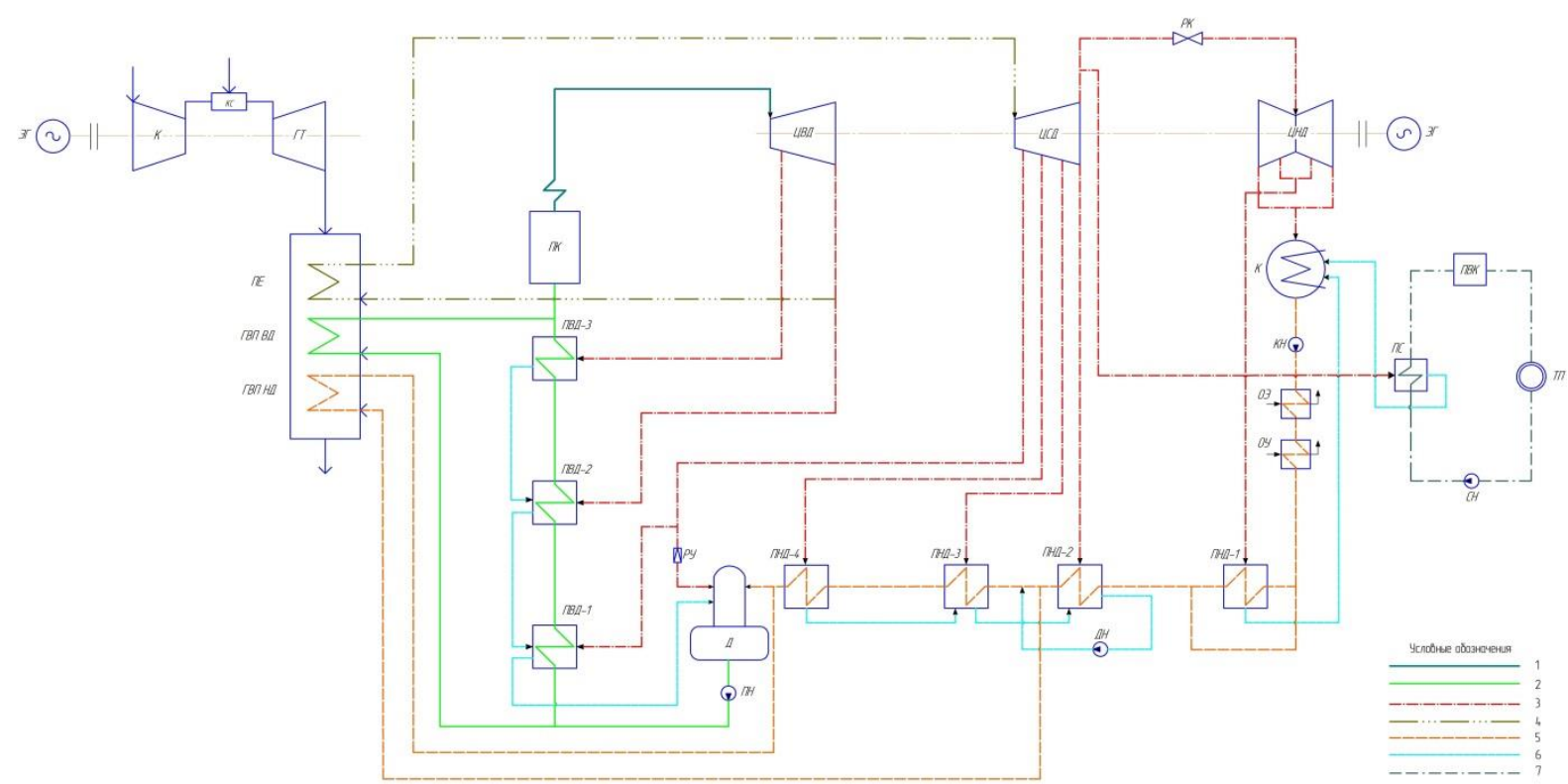

Рис. 1. Принципиальная тепловая схема разрабатываемой ПГУ: 1 - острый пар; 2 - питательная вода; 3 - пар отборов; 4 - пар промежуточного перегрева; 5 - основной конденсат; 6 - дренаж; 7 -сетевая вода

Fig. 1. Principal thermal scheme of the developed combined cycle power plant (CCPP): 1 - main steam; 2 - feed water; 3 -extraction steam; 4 -reheated steam; 5 - main condensate; 6 -drainage water; 7 -delivery water

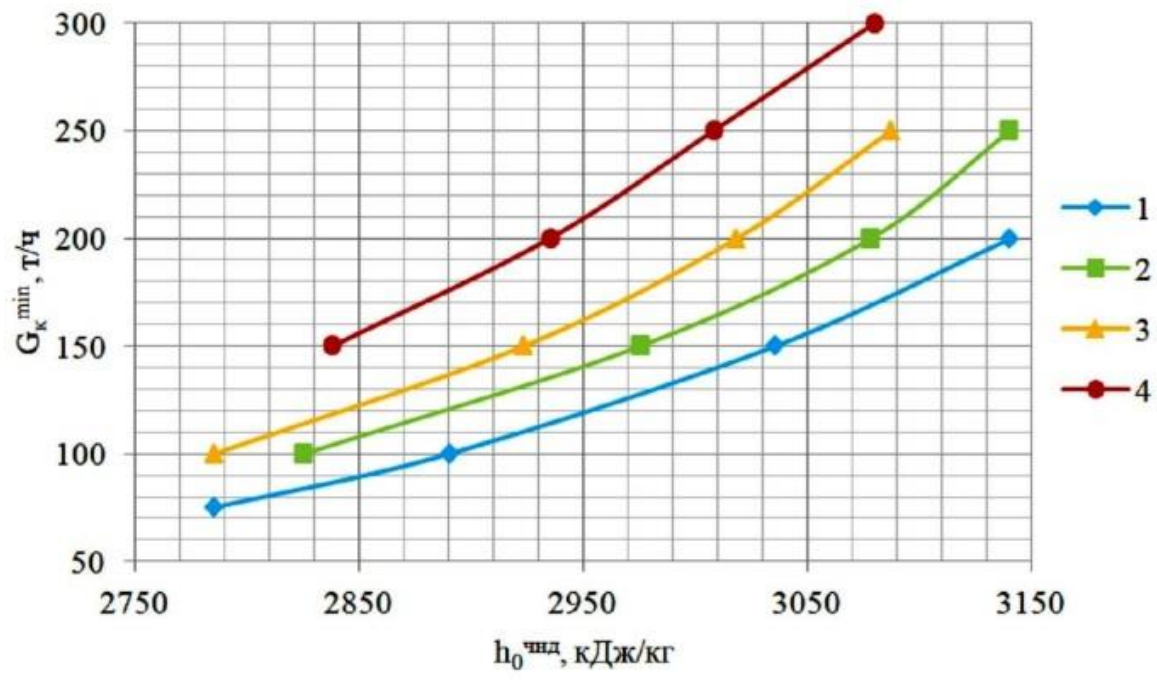

Pис. 2. Зависимость минимального расхода пара в конденсатор турбины $K-210-130$, необходимого для охлаждения последних ступеней, от энтальпии перед ЧНД при разных давлениях в конденсаторе $P_{k}$, МПа: 1) 0,0035; 2) 0,004 ; 3) 0 ,0045; 4) 0,006

Fig. 2. Dependence of the minimum steam consumption to the condenser of steam turbine $K-210-130$ required for cooling the last stages on the enthalpy before low-pressure section at different pressures in the condenser $\left.P_{c}, M P a: 1\right) 0,0035$, 2) 0,$004 ; 3) 0,0045 ; 4) 0,006$

В качестве исходного для анализа режима принят конденсационный режим работы турбины К-210-130 c номинальной электрической мощностью ПТ $N_{9}{ }^{\mathrm{mT}}=210 \mathrm{MBT}$, начальных параметрах пара $-P_{0}=12,8 \mathrm{MПа}$, $t_{0}=540{ }^{\circ} \mathrm{C}$, температуре перегретого пара $t_{\text {пा }}=540{ }^{\circ} \mathrm{C}$, давлении в конденсаторе $-P_{\mathrm{K}}=0,0039$ МПа и паспортных значениях давлений пара в регенеративных отбоpax. Для этого режима расход острого пара на турбоустановку (ТУ) $G_{0}=169,8$ кг $/ \mathrm{c}$, удельный расход условного топлива (УРУТ) по выработке электроэнергии $b_{9}{ }^{\text {cт }}{ }^{\sigma \mathrm{p}}=315$ г у.т./(кВт·ч). В дальнейших исследованиях теплофикационных режимов расход острого пара на турбоустановку принимается постоянным $-G_{0}=169,8$ кг $/ \mathrm{c}$.

Для определения показателей работы прототипа при переводе К-210-130 в теплофикационный режим традиционным способом и схемы ПГУ в теплофикационном режиме принят температурный график теплосети $-t_{\text {пс }} / t_{\mathrm{oc}}=150 / 70{ }^{\circ} \mathrm{C}$ (для г. Томска), коэффициент теплофикации $-\alpha_{\text {тэц }}=0,55$. Расчеты выполняются для режимов максимального отпуска теплоты из регулируемого отбора при заданном в нем давлении $P_{\text {отб }}=0,12$ МПа (для выбранного климатического района соответствует температуре прямой сети при 
включении пиковой водогрейной котельной (ПВК) $\left.\left(t_{\mathrm{HB}}=-15^{\circ} \mathrm{C}\right)\right)$ с сохранением минимально необходимого для охлаждения последних ступеней турбины расхода пара в конденсатор.

Определение показателей тепловой экономичности прототипа при переводе К-210-130 в теплофикационный режим традиционным способом выполняется при $t_{\text {пा }}=540{ }^{\circ} \mathrm{C}$ и $G_{\mathrm{\kappa}}{ }^{\mathrm{min}}=30 \mathrm{\kappa r} / \mathrm{c}$ (определен по рис. 2 ). Этому режиму соответствуют следующие показатели работы ПТУ: электрическая мощность ПТ $N_{\ni}{ }^{\text {пा }}=164,7$ МВт; тепловая мощность отопительного отбора $Q_{\mathrm{T}}=208,6 \mathrm{MBт}$; УРУТ по выработке электроэнергии (здесь и далее определен по физическому методу) $b_{9}{ }^{\text {ㄷ бр }}=230$ г у.т. $/($ кВт ч) $)$.

Расчет тепловой схемы ПГУ выполняется при работе ПТУ по тепловому графику при температуре наружного воздуха $t_{\text {нв }}=-15^{\circ} \mathrm{C}$ при разных значениях температуры промежуточного перегрева пара с целью определения наибольшей эффективности в зимний период. Максимальное значение температуры ПП принято равным номинальному значению $t_{\text {mा }}=540{ }^{\circ} \mathrm{C}$. Минимальная величина $t_{\text {пा }}$ ограничена температурой пара за ЦВД ПТ. Снижение температуры ПП приводит к уменьшению $G_{\mathrm{\kappa}}{ }^{\mathrm{min}}$, что при неизменном расходе острого пара вызывает рост тепловой нагрузки.

Для рассматриваемой ГТ-надстройки в качестве прототипа ГТУ [19] принята Siemens SGT-1000F $[20,21]$. Используя параметры и характеристики газотурбинного цикла этой установки, был выполнен расчет схемы ГТУ при температуре наружного воздуха на входе в компрессор $t_{0 \mathrm{k}}=+15{ }^{\circ} \mathrm{C}$, температуре газов на входе в газовую турбину $t_{0 \mathrm{r}}=1190^{\circ} \mathrm{C}$ и степени повышения давления в компрессоре $\varepsilon=15,6$ [21]. Расход газов $G_{\mathrm{r}}=281 \mathrm{\kappa} / \mathrm{c}$ в этом режиме определен из совместного расчета ГТУ и ПТУ при работе паровой турбины в конденсационном режиме. Этот режим в дальнейшем принят за исходный для ГТУ: электрическая мощность $Г Т N_{9}{ }^{\mathrm{IT}}=98,6 \mathrm{MBT}$, температура газов на выходе из $\Gamma \mathrm{T} t_{\mathrm{r}}=589^{\circ} \mathrm{C}$, электрический коэффициент полезного действия (КПД) ГТ $\eta_{\ni}{ }^{\text {гт }} \mathrm{p}=0,368$, удельный расход условного топлива по выработке ЭЭ ГТ $b_{9}{ }^{\mathrm{rT}}{ }^{\mathrm{p}}=349$ г у.т. $/(\mathrm{\kappa BT} \cdot ч)$. Для данного режима показатели работы ПГУ: расход пара на турбоустановку $G_{0}=133,0$ кг $/ \mathrm{c}$, электрическая мощность $\Pi \mathrm{N} N_{\ni}^{\mathrm{m}}=189,4 \mathrm{MBT}$, электрическая мощность ПГУ $N_{\text {эㅍ }}^{\text {пा }}=288,0$ МВт, УРУТ по выработке электроэнергии $b_{9}^{\text {пाу бр }}=272$ г у.т./(КВт·ч). Температура ПП равна номинальной $t_{\text {пा }}=540{ }^{\circ} \mathrm{C}$. Расход пара в конденсатор соответствует номинальному конденсационному режиму ПТ $G_{\mathrm{K}}=115,5$ кг $/ \mathrm{c}$.

Для определения показателей работы ГТУ при $t_{\mathrm{HB}}=-15{ }^{\circ} \mathrm{C}$ использовались результаты расчетного режима ГТУ при $t_{\mathrm{HB}}=+15{ }^{\circ} \mathrm{C}$ и универсальные зависимости изменения основных параметров стационарных энергетических ГТУ по отношению к их значениям по ISO от температуры наружного воздуха [20, рис. 6.25]. Расход газов и воздуха принят одинаковым. Влияние охлаждения учтено снижением электрического КПД ГТ на $2 \%$. С учетом вышесказанного для режима при $t_{\mathrm{HB}}=-15{ }^{\circ} \mathrm{C}$ показатели работы ГТУ:
$G_{\mathrm{r}}=306 \kappa \Gamma / \mathrm{c}, N_{\ni}{ }^{\mathrm{rT}}=113,9 \mathrm{MBT}, t_{\mathrm{r}}=580{ }^{\circ} \mathrm{C}, \eta_{9}{ }^{\mathrm{rT}}{ }^{\mathrm{T}}=0,376$,

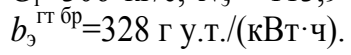

Температура уходящих газов (на выходе из КУ) $t_{\text {ух }}$ изначально принимается равной $120{ }^{\circ} \mathrm{C}$. Параметры тепловой схемы в переменном режиме определяются по общеизвестным зависимостям Стодолы-Флюгеля для ПТ и поверочного расчета теплообменников. КПД проточной части ПТ для частей высокого и среднего давления принимались постоянными при неизменном расходе острого пара, КПД ЧНД оценивался в зависимости от объемного пропуска пара. Во всех режимах расход питательной воды пропускается через поверхности ГВП ВД, минуя подогреватели высокого давления (ПВД). Необходимый расход основного конденсата поступает в ГВП НД после второго подогревателя низкого давления (ПНД-2) с целью предотвращения низкотемпературной коррозии в котле-утилизаторе. Температура ПВ на входе в паровой котел (ПК) равна номинальному значению и не меняется в вариантных расчетах, температура ОК на входе в деаэратор также постоянна и соответствует подогреву воды в деаэраторе для конденсационного режима работы ПТУ.

\section{Выбор оптимальной температуры промежуточного перегрева пара при работе ПгУ в теплофикационном режиме}

В качестве показателей тепловой экономичности ПГУ $[1,21]$ при анализе приняты удельный расход условного топлива по выработке электроэнергии (по физическому методу) (1) и коэффициент использования теплоты топлива (КИТТ) (2).

Удельный расход условного топлива по выработке электроэнергии

$$
b_{\ni}^{\text {пгу бр }}=\frac{B_{\ni}^{\text {пгу }} \cdot 3600}{N_{\ni}^{\text {пгу }}}, \text { г У.т./(кВт·ч), }
$$

где $B_{\ni}^{\text {пу }}$, кг у.т./с - расход условного топлива (УТ) по выработке электроэнергии ПГУ; $N_{э}^{\text {пгу }}$ МВт - электрическая мощность ПГУ.

Коэффициент использования теплоты топлива

$$
K И T T=\frac{N_{\mathrm{g}}^{\mathrm{nry}}+Q_{\mathrm{r}}}{Q_{\mathrm{n \kappa}}^{0}+Q_{\mathrm{rc}}}
$$

где $Q_{\mathrm{T}}$, МВт - тепловая мощность отопительного отбора; $Q_{\text {пा }}^{0}$, МВт - теплота, выделившаяся при сжигании УТ в ПК; $Q_{\text {кс, }}$ МВт - теплота, подведенная в камере сгорания ГТУ.

Результаты расчета представлены в табл. 1.

Оптимальная температура промежуточного перегрева пара в котле-утилизаторе ПГУ в теплофикационном режиме составляет $510{ }^{\circ} \mathrm{C}$, что соответствует наименьшему значению УРУТ по выработке электроэнергии $b_{9}^{\text {пгу бр }}=185$ г у.т./(кВт.ч). Снижение значения этого показателя достигает 19,5 \% по сравнению с энергоблоком T-165/210-130 при работе в теплофикационном режиме (рис. 3). Оптимальное значение $t_{\text {пा }}$ подтверждается максимальным КИТТ - 0,775. Для энергоблока Т-165/210-130 КИТТ ниже на 11,5 \% и составляет 0,695 (рис. 3 ). 
Таблица 1. Результаты расчета тепловой схемы ПГУ при разных значениях температуры промежуточного перегрева пара в КУ при работе в теплофикачионном режиме

Table 1. Calculation results of CCPP thermal scheme at different values of steam reheating temperature in heat recovery steam generator (HRSG) at operation in heat-extraction mode

\begin{tabular}{|c|c|c|c|c|c|c|c|}
\hline \multicolumn{8}{|c|}{ Исходные данные/Source data } \\
\hline$t_{\mathrm{mI}},{ }^{\circ} \mathrm{C} / t_{\mathrm{sr}},{ }^{\circ} \mathrm{C}$ & 540 & 520 & 510 & 500 & 490 & 480 & 460 \\
\hline$G_{\mathrm{K}}{ }^{\min }, \mathrm{\kappa} \Gamma / \mathrm{c} / G_{\mathrm{c}}{ }^{\min }, \mathrm{kg} / \mathrm{s}$ & 27,0 & 24,0 & 22,9 & 21,2 & 20,3 & 19,2 & 18,0 \\
\hline$t_{\mathrm{yx}}{ }^{*},{ }^{\circ} \mathrm{C} / t_{\mathrm{wg}}{ }^{*},{ }^{\circ} \mathrm{C}$ & 120,0 & 120,0 & 120,0 & - & - & - & - \\
\hline \multicolumn{8}{|c|}{ Результаты расчета/Calculation results } \\
\hline$t_{\mathrm{yx}},{ }^{\circ} \mathrm{C} / t_{\mathrm{wg}},{ }^{\circ} \mathrm{C}$ & - & - & - & 130,4 & 140,9 & 151,5 & 172,4 \\
\hline$Q_{\mathrm{T}}, \mathrm{MBT} / Q_{\mathrm{t}}, \mathrm{MW}$ & 286,9 & 296,6 & 300,3 & 302,5 & 302,7 & 303,3 & 302,1 \\
\hline$Q_{\mathrm{K}}, \mathrm{MBT} / Q_{\mathrm{c}}, \mathrm{MW}$ & 67,3 & 60,2 & 57,4 & 53,4 & 51,1 & 48,7 & 45,5 \\
\hline$N_{\mathrm{s}}^{\mathrm{nT}}, \mathrm{MBT} / N_{\mathrm{e}}^{\mathrm{st}}, \mathrm{MW}$ & 180,7 & 176,9 & 175,3 & 172,9 & 171,0 & 168,7 & 165,2 \\
\hline$N_{\mathrm{s}}^{\text {nry }}, \mathrm{MB} / N_{\mathrm{e}}^{\text {ccpp }}, \mathrm{MW}$ & 294,7 & 290,8 & 289,2 & 286,8 & 284,9 & 282,6 & 279,1 \\
\hline КИТТ/FHUF & 0,766 & 0,773 & 0,775 & 0,774 & 0,772 & 0,769 & 0,764 \\
\hline 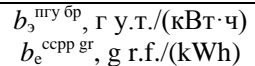 & 188 & 186 & 185 & 186 & 187 & 188 & 191 \\
\hline
\end{tabular}

*При низких температурах перегретого пара $t_{y x}$ не может быть выдержана на уровне $120^{\circ} \mathrm{C}$ и определяется расчетом.

*At low temperatures of reheated steam $t_{w g}$ cannot be maintained at $120^{\circ} \mathrm{C}$ level and is determined by calculation.

Электрическая мощность ТЭС $N_{\ni}^{\text {пгу }}$ за счет ГТ-надстройки составляет 279-295 МВт во всем диапазоне температур ПП (рис. 4). При уменьшении $t_{\text {пп }}$ падает электрическая мощность паровой турбины $N_{\ni}^{\text {пт }}$ и потери тепла в конденсаторе $Q_{\text {к. }} N_{\ni}^{\text {пт }}$ меняется от 180 до 165 МВт при изменении $t_{\text {пп }}$ от 540 до $460{ }^{\circ} \mathrm{C}$, а $Q_{\kappa}$ - от 67 до 45 МВт. При оптимальной температуре ПП электрическая мощность ПТ составляет 175 МВт. Этот результат представляет особенный интерес, так как электрическая мощность паровой турбины в новых условиях работы при значении температуры промежуточного перегрева существенно ниже номинального значения, превышает мощность исходного энергоблока Т-165/210-130 на 10 МВт.
Следует отметить, что на значение $N_{\ni}^{\text {пт }}$, наряду со снижением $G_{\mathrm{K}}^{\min }$ при уменьшении $t_{\text {пп }}$, также оказывает влияние на изменение относительного расхода воды через группу регенеративных подогревателей, вызывающее изменение расхода пара в регенеративные отборы паровой турбины $\Sigma G_{\text {рег }}$.

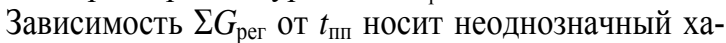
рактер из-за необходимости во всех режимах поддерживать заданную температуру ОК на входе в деаэратор. При заданном расходе острого пара $G_{0}$ при понижении $t_{\text {пп }}$ до $510{ }^{\circ} \mathrm{C}$ сокращается расход пара в отборы на ПНД ПТ из-за увеличения тепловой мощности поверхности ГВП НД в связи с повышением температуры газов после ПП КУ. Падение расхода пара на ПНД одновременно со снижением $G_{\kappa}$ min дополнительному росту расхода пара в отопительный отбор $G_{\text {т }}$ (рис. 5), что усиливает эффект использования ГТ-надстройки в теплофикационном режиме.


(рис. 4) по сравнению с энергоблоком Т-165/210-130. В этом режиме происходит полное вытеснение ПНД-3 и ПНД-4 рассматриваемой ТУ.

Дальнейшее уменьшение $t_{\text {пп }}$ приводит к неполному использованию теплоты уходящих газов в КУ, соответственно, растут потери с уходящими газами из-за повышения их температуры (рис. 4). Для поддержания заданной температуры ОК перед деаэратором (как было сказано раньше) приходится прибегать к снижению расхода ОК через ГВП НД, что влечет незначительное


ратуре ПП ниже $510{ }^{\circ} \mathrm{C}$ прирост тепловой мощности отопительного отбора ТУ замедляется.

C понижением температуры перегретого пара происходит все большее вытеснение регенерации низкого давления, вплоть до того, что при значениях $t_{\text {пп }}$ ниже $510{ }^{\circ} \mathrm{C}$ происходит неполное использование тепла в котле-утилизаторе, растет температура уходящих газов, соответственно, растут потери с уходящими газами (рис. 4).

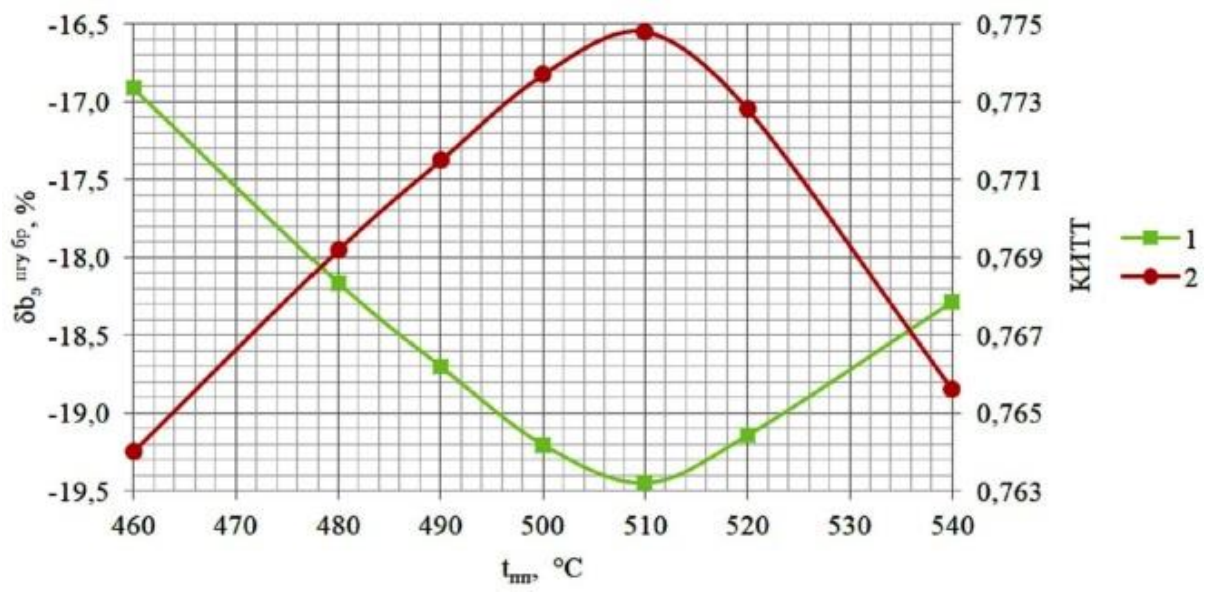

Pис. 3. Снижение УРУТ по выработке электроэнергии по сравнению с энергоблоком T-165/210-130 и изменение КИТТ в зависимости от температуры промежуточного перегрева пара при работе ПГУ в теплофикачион-

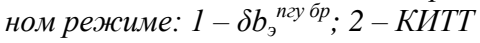

Fig. 3. Decrease in specific reference fuel consumption for electricity generation in comparison with T-165/210-130 energy unit and dependence of fuel heat utilization factor on steam reheating temperature at operation of CCPP in heatextraction mode: $1-\delta b_{e}^{\text {ccpp gr }^{2}} ; 2$-fuel heat utilization factor (FHUF) 


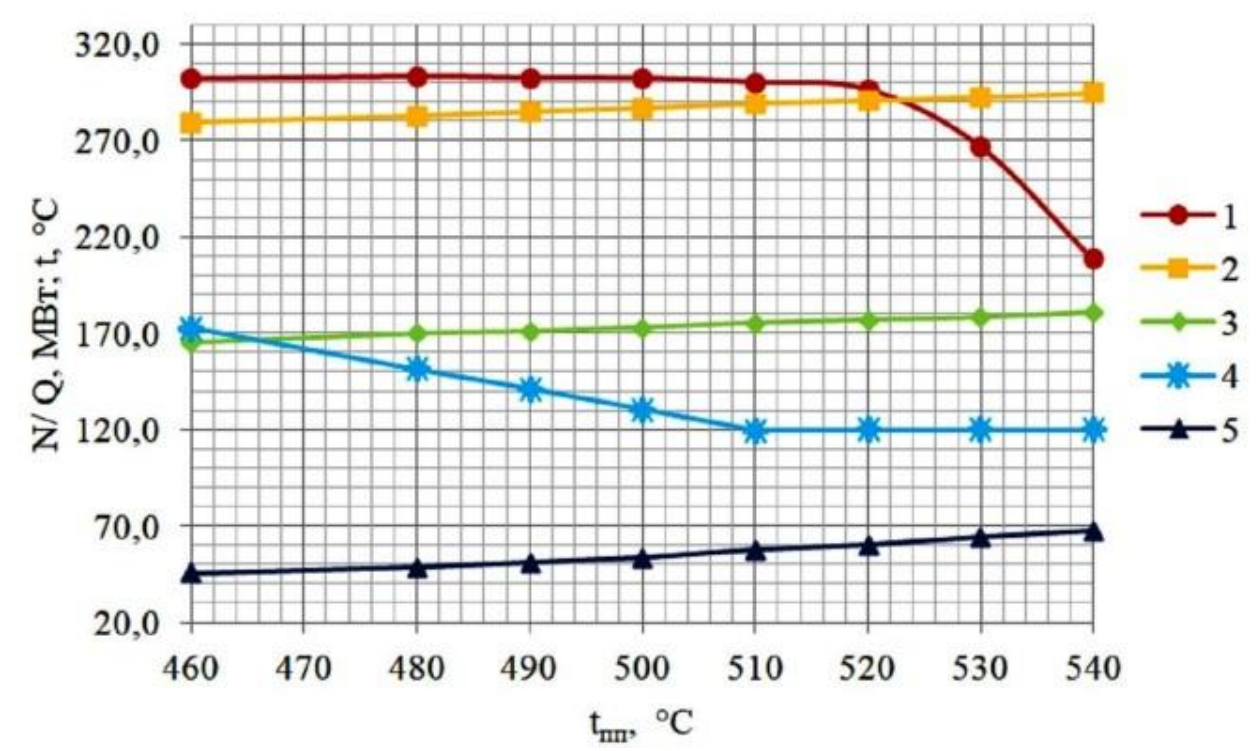

Рис. 4. Зависимость показателей работы ПГУ в теплофикационном режиме от температуры промежуточного перегрева пара: $1-Q_{m} ; 2-N_{\ni}^{n z y} ; 3-N_{\ni}^{n m} ; 4-t_{y x} ; 5-Q_{\kappa}$

Fig. 4. Dependence of CCPP performance at operation in heat-extraction mode on steam reheating temperature: $1-Q_{t} ; 2-$ $N_{e}^{c c p p} ; 3-N_{e}^{s t} ; 4-t_{w g} ; 5-Q_{c}$

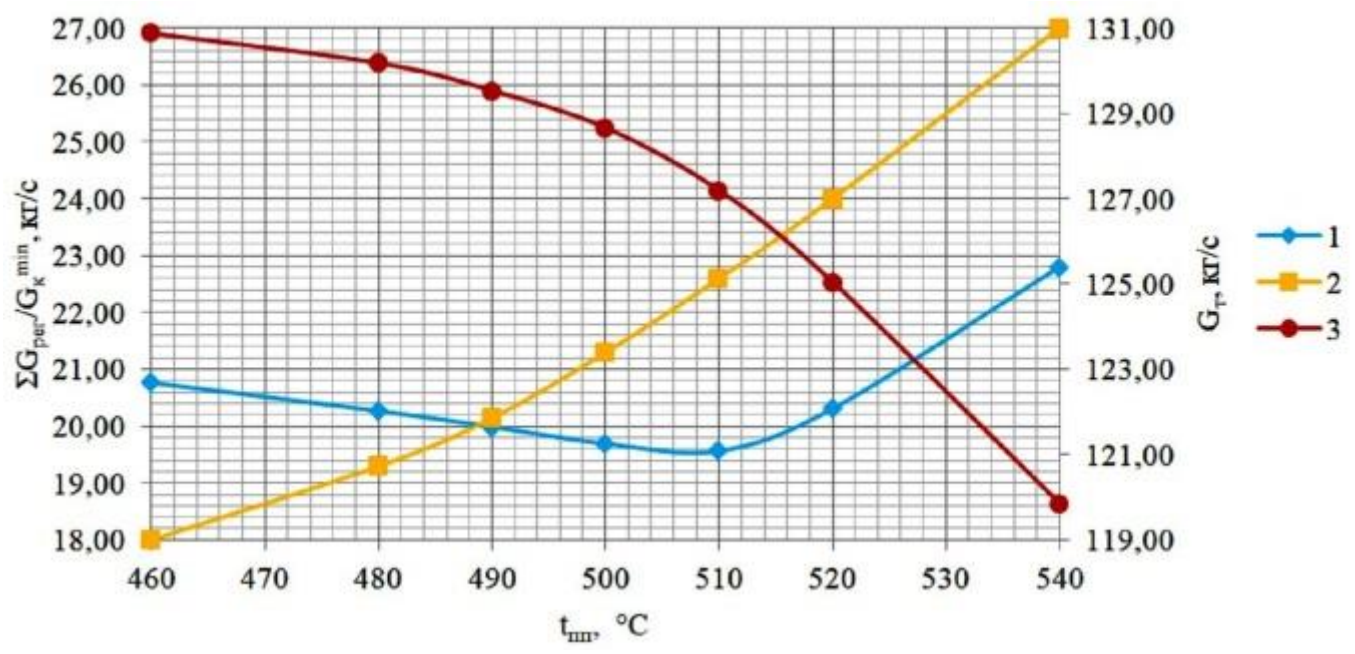

Рис. 5. Зависимость суммарного расхода пара на регенерацию, минимально необходимого для охлаждения последних ступеней турбины расхода пара в конденсатор и расхода пара в отопительный отбор, от температуры промежуточного перегрева пара при работе ПГУ в теплофикачионном режиме: $1-\Sigma G_{\text {рег }} 2-G_{\kappa}{ }^{\min } ; 3-G_{m}$

Fig. 5. Dependence of the total steam consumption for regeneration, minimum steam consumption to the condenser required for cooling the last stages of steam turbine and the steam consumption to heating extraction on steam reheating temperature at operation of CCPP in heat-extraction mode: $1-\Sigma G_{\text {reg }} ; 2-G_{c}{ }^{\mathrm{min}} ; 3-G_{t}$

Сравнение результатов расчета схемы Пгу с энергоблоком T-165/210-130 в характерных режимах работы

В теплофикационном режиме при оптимальной температуре ПП $t_{\text {пा }}=510^{\circ} \mathrm{C}$ благодаря ГТ-надстройке электрическая мощность повышается не только за счет ГТУ, но и из-за увеличения мощности самой ПТ (на $6 \%$ по сравнению с исходным вариантом при работе в теплофикационном режиме при $t_{\text {пा }}=540^{\circ} \mathrm{C}$ ).

Прирост $N_{\ni}^{\text {Пгу }}$ относительно исходной схемы ПТУ в конденсационном режиме при номинальной температуре ПП - $37 \%$, в теплофикационном режиме при оптимальной температуре ПП - 76 \% (рис. 6).
Эффективность использования ГТ-надстройки, помимо роста электрической мощности станции на величину мощности газовой турбины, заключается в повышении выработки электроэнергии на тепловом потреблении паровой турбиной по сравнению с исходной схемой парового энергоблока за счет возрастания тепловой мощности регулируемого отбора изза сокращения расхода пара в конденсатор и на регенеративные подогреватели турбины.

Для ПГУ в конденсационном режиме с номинальной температурой ПП $b_{\ni}{ }^{\text {пгу бр }}$ снижается на $13 \%$, в теплофикационном с оптимальной температурой ПП - на 19 \% по сравнению с энергоблоком Т-165/210-130. 
Тепловая мощность отопительного отбора турбоустановки в составе ПГУ выше, чем исходной схемы ПТУ в теплофикационном режиме, на 90 МВт (за счет снижения минимально необходимого для охлаждения последних ступеней турбины расхода пара в конденсатор из-за понижения температуры ПП и вытеснения регенерации в КУ при сохранении расхода пара на турбину).

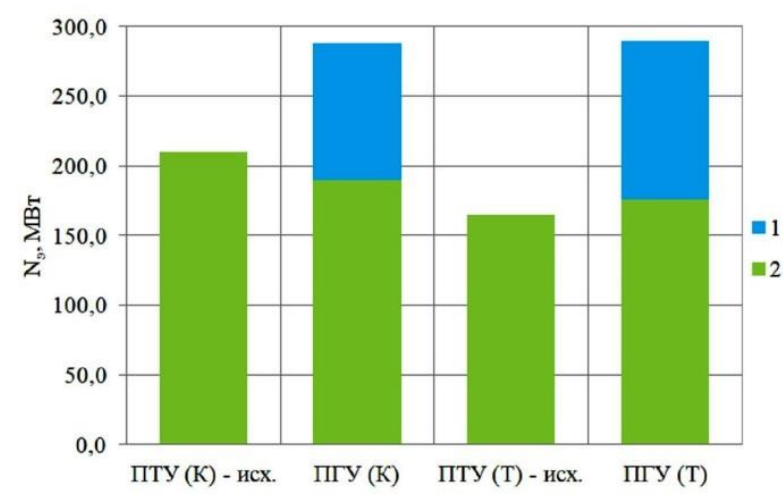

Pис. 6. Прирост электрической мощности ТЭС за счет ГТ-надстройки в конденсационном и теплофикачионном режимах по сравнению с энергоблоком T-165/210-130: $1-N_{\ni}^{2 m} ; 2-N_{\ni}{ }^{n m}$

Fig. 6. Increase in electric power of thermal power plant due to the GT-topping in condensing and heatextraction modes compared with T-165/210-130 energy unit: $1-N_{e}^{g t} ; 2-N_{e}^{s t}$

\section{Анализ ключевых вопросов, связанных с организацией} промежуточного перегрева в КУ ГТ-надстройки

Вытеснение ПП в КУ требует решения вопроса об использовании поверхности промежуточного пароперегревателя парового котла для других целей. Одним из возможных является вариант применения данной поверхности для нагрева циклового воздуха ГТУ. Вопросы реконструкции парового котла в данной работе не рассматриваются, однако предполагается ее возможность [22], поэтому КПД котла сохраняется без изменений.

Вытеснение паровой регенерации может привести к перегрузке отдельных ступеней и отсеков в отдельных режимах при сохранении расхода пара на турбину, равным номинальному значению. В расчетах конденсационного режима расход пара через последнюю ступень турбины не превышал максимально допустимого значения с соответствующим понижением $G_{0}$. В теплофикационном режиме расчет схемы выполнялся при номинальном значении расхода острого пара. Результаты расчета этого режима показывают, что рост паровой нагрузки на отдельные отсеки не приводит к повышению давления в камере регулирующей ступени выше максимально допустимого для прототипа. Вопросы надежности работы ступеней ЦВД наиболее просто решаются для турбин, проектируемых с запасом прочности с целью работы при отключенных ПВД, в противном случае для подтверждения надежности работы отдельных ступеней тре- буются расчеты на прочность и при необходимости должна выполняться реконструкция проточной части либо рекомендации по снижению расхода острого пара. В последнем случае энергетический эффект снижается и требует пересчета.

Недостатком предложенного схемного решения можно считать проблему, возникающую с промежуточным перегревом пара при отключениях ГТУ в надстроенном энергоблоке, что будет приводить его к останову. Требуется решение вопроса резервирования оборудования.

\section{Выводы}

1. Эффективность работы теплофикационного парового энергоблока с промежуточным перегревом может быть повышена путем осуществления промежуточного перегрева в котле-утилизаторе ГТ-надстройки.

2. Вариант ГТ-надстройки с вытеснением промежуточного перегрева пара из парового котла и частично регенерации турбоустановки Т-165/210-130 в котел-утилизатор позволяет увеличить в конденсационном режиме электрическую мощность на 37 \% по сравнению с исходным паротурбинным энергоблоком. Температура ПП сохраняется равной номинальному значению.

3. Наивысшая экономичность ПГУ с вытеснением промежуточного перегрева пара в КУ при работе по тепловому графику с максимальным отпуском теплоты из регулируемого отбора при номинальном давлении пара в отопительном отборе 0,12 МПа с сохранением минимально необходимого для охлаждения последних ступеней турбины расхода пара в конденсатор соответствует $t_{\text {mा }}=510{ }^{\circ} \mathrm{C}$, что значительно ниже номинального значения $\left(540{ }^{\circ} \mathrm{C}\right)$. Показатели работы ПГУ:

- электрическая мощность ПГУ - 289 МВт;

- электрическая мощность ПТ - 175 МВт (выросла на $6 \%$ по сравнению с работой ПТУ в теплофикационном режиме без ГТ-надстройки);

- отпуск тепла из отопительного отбора порядка 300 МBт;

- УРУТ по выработке ЭЭ - 185 г у.т./(кВт·ч);

- коэффициент использования теплоты топлива $-0,775$.

4. Модернизация ТЭС с использованием ГТ-надстройки с котлом-утилизатором для промежуточного перегрева пара и частичного вытеснения регенерации позволит в отопительный период по сравнению с исходной схемой энергоблока повысить:

- электрическую мощность станции на 76 \%;

- отпуск теплоты из отопительного отбора внешнему потребителю на 45 \%;

- КИТТ на $11,5 \%$.

5. Предложенный вариант модернизации позволит снизить УРУТ по выработке электроэнергии в летнем режиме на $13 \%$, в зимнем - на $19 \%$. 


\section{СПИСОК ЛИТЕРАТУРЫ}

1. Combined Cycle Gas and Steam Turbine Power Plants / R. Kehlhofer, F. Hannemann, F. Stirnimann, B. Rukes. - Tulsa, Oklahoma, USA: PennWell Corporation, 2009. - 434 p.

2. Бурмистров Н.А., Осипов Р.А. Перспективные направления перевооружения действующих ТЭС (тепловых электростанций) на базе газовых надстроек // Электроэнергетика глазами молодежи: Материалы VIII Международной научнотехнической конференции. - Самара, 2017. - С. 112-113.

3. Scherer V., Scherer D. The Gas turbine GT26 in combined cycle application: conversion of a coal power plant into a modem combined cycle firing natural gas and oil. - Birmingham: International Gas Turbine Institute/American Society of Mechanical Engineers, 1996. - $10 \mathrm{p}$.

4. Березинец П.А. Газотурбинная надстройка блока 300 МВт Костромской ГРЭС (государственной районной электростанции) // Электрические станции. - 1999. - № 7. - С. 64-72.

5. Economic efficiency of a gas-turbine topping for steam reheating at heating turbo-installations / O.Yu. Romashova, L.A. Belyaev, A.A. Tubolev, E.A. Skrebatun // MATEC (Materials science, Engineering and Chemistry) Web of Conferences. - 2017. V. $91 .-5 \mathrm{p}$.

6. Заугольникова Я.А., Ромашова О.Ю., Скребатун Е.А. Экономичность использования газотурбинной надстройки на энергоблоках тепловых электростанций // Интеллектуальные энергосистемы: Труды III Международного молодежного форума. - Томск, 2015. - Т. 2. - С. 107-110.

7. Efficiency of steam reheating in heat recovery steam generator at thermal power plant with gas turbine topping / O.Yu. Romashova, A.A. Minor, V.N. Martyshev, A.A. Tubolev // MATEC (Materials science, Engineering and Chemistry) Web of Conferences. 2017. - V. $110 .-8$ p.

8. Development of natural gas fired combined cycle plant for trigeneration of power, cooling and clean water using waste heat recovery: techno-economic analysis / G. Mohan, S. Dahal, U. Kumar, A. Martin, H. Kayal // Energies. - 2014. - № 7. P. 6358-6381.

9. Karaagac M.O., Kabul A., Yigit F. Performance analysis of Natural Gas Combined Cycle Power Plant // Journal of Polytechnic - Politeknik Dergisi. - 2019. - V. 22. - P. 319-325.

10. Selection of optimal reheat temperature in heat recovery boiler at combined heat and power plant with gas turbine topping / O.Yu. Romashova, A.A. Tubolev, A.A. Matveeva, A.V. Zenkov // MATEC (Materials science, Engineering and Chemistry) Web of Conferences. -2017 . - V. 141. $-4 \mathrm{p}$.

11. Особенности схемных решений паротурбинных установок $3 А О$ (закрытого акционерного общества) «Уральский турбинный за- вод», входящих в состав ПГУ (парогазовых установок) / А.А. Гольдберг, Т.Л. Шибаев, Х.К. Панэке Агилера, М.Ю. Степанов // Теплоэнергетика. - 2013. - № 8. - С. 27-31.

12. Khan M.N., Tlili I. New advancement of high performance for a combined cycle power plant: thermodynamic analysis // Case Studies in Thermal Engineering. - 2018. - № 12. - P. 166-175.

13. The optimum performance of the combined cycle power plant: a comprehensive review / T.K. Ibrahim, M.K. Mohammed, O.I. Awad, M. Rahman, G. Najafi, F. Basrawi, A.N.A. Alla, R. Mamat // Renewable and Sustainable Energy Reviews. 2017. - V. 79. - P. 459-474.

14. Javadi M.A., Ghomashi H. Thermodynamics analysis and optimization of Abadan combined cycle power plant // Indian Journal of Science and Technology. - 2016. - V. 9. - № 7. - 12 p.

15. Thermodynamic evaluation of combined cycle plants / N. Woudstra, T. Woudstra, A. Pirone, T. van der Stelt // Energy Conversion and Management. - 2010. - V. 51. - № 5. - P. 1099-1110.

16. Соболев С.П. Паровая турбина К-160-130 ХТГЗ (Харьковского турбогенераторного завода). - М.: Энергия, 1980. - 192 с.

17. Балабанович В.К. Охлаждение проточной части паровых турбин на режимах с минимально-вентиляционными пропусками пара // Известия вузов. Энергетика. - 1981. - № 11. C. 105-107.

18. Проведение эксплуатационных испытаний турбины Т-178/210-130 ст. № 12 с целью определения фактической экономичности и корректировки диаграммы режимов ОАО (открытое акционерное общество) «Сургутская ГРЭС-1» (государственная районная электростанция): технический отчет / ОАО (открытое акционерное общество) «Инженерный центр энергетики Урала» - предприятие «УралОРГРЭС» (организация и рационализация государственных районных электростанций и сетей). - Екатеринбург, 2008. - 97 с.

19. Ibrahim T.K., Rahman M.M., Abdalla A.N. Gas turbine configuration for improving the performance of combined cycle power plant // Procedia Engineering. - 2011. - V. 15. - P. 42164223.

20. Газотурбинные энергетические установки / С.В. Цанев, В.Д. Буров, А.С. Земцов, А.С. Осыка. - М.: ИД Московского энергетического института, 2011. $-428 \mathrm{c}$.

21. Трухний А.Д. Парогазовые установки электростанций. - М.: ИД Московского энергетического института, 2013. - $648 \mathrm{c.}$

22. Ольховский Г.Г., Чернецкий Н.С., Березинец П.А. Модернизация энергетических блоков путем их надстройки газовыми турбинами // Электрические станции. - 1997. - № 4. - С. 9-18.

Поступила 12.09.2019 г.

\section{Информация об авторах}

Минор A.A., аспирант НОЦ И.Н. Бутакова Инженерной школы энергетики Национального исследовательского Томского политехнического университета.

Ромашова О.Ю., кандидат технических наук, доцент НОЦ И.Н. Бутакова Инженерной школы энергетики Национального исследовательского Томского политехнического университета. 
UDC 621.311.22.002.5:621.181.27.013.3:621.438

\title{
EFFICIENCY ANALYSIS OF STEAM REHEATING IN HEAT RECOVERY STEAM GENERATOR OF HEAT-EXTRACTION ENERGY UNIT GT-TOPPING
}

\author{
Alexander A. Minor ${ }^{1}$, \\ aam45@tpu.ru \\ Olga Yu. Romashova ${ }^{1}$, \\ roma@tpu.ru \\ 1 National Research Tomsk Polytechnic University, \\ 30, Lenin avenue, Tomsk, 634050, Russia.
}

Relevance. Redeveloping of the existing heat-power equipments is one of the priority areas of power generation development. Adoption of gas turbine unit to the thermal scheme of steam-power energy unit with the preservation of power plant infrastructure is a less costly variant comparing with the construction of new combined cycle power plants energy units. One of the low-cost variant for the integration of gas turbine units into the thermal scheme of steam-turbine energy units is to use the heat recovery steam generator thermal capacity for reheating the working fluid spent in the steam turbine with utilization of the remaining heat for feed water and main condensate heating. Compared with the most common schemes with displacement of regenerative heating to heat recovery steam generator, the proposed alternative variant allows increasing the thermal efficiency of the steam-turbine circuit. The analysis of gas turbine units energy characteristics showed the need to research the scheme and parameters of the steam reheating for heat-extraction energy units taking into account the operating modes of steam turbine.

Object: steam-turbine energy unit T-165/210-130 with gas turbine topping (GT-topping) for reheating of the working fluid spent in the steam turbine with utilization of the remaining heat for feed water and main condensate heating.

The aim of the research is to select scheme and calculate the optimal parameters of steam reheating as applied to the steam-turbine energy unit T-165/210-130 with GT-topping, analyze its main performances during the work on the heat schedule when steam reheating temperature changes in heat recovery steam generator.

Methods: system analysis and mathematical modeling.

Results. The authors have developed the mathematical model and program for calculating the proposed scheme and performed the features analysis associated with the organization of steam reheating in GT-topping heat recovery steam generator. Furthermore, we showed that the efficiency of heat-extraction energy unit with steam reheating can be increased by organizing steam reheating in GTtopping heat recovery steam generator. In addition, we determined that in this case, the highest efficiency of a steam-turbine energy unit with GT-topping during operation in the design heat-extraction mode is achieved, when steam reheating temperature is below the nominal value.

\section{Key words:}

Steam reheating, combined cycle power plant, gas turbine topping, heat recovery steam generator, heat-extraction mode, specific fuel consumption, fuel heat utilization factor, steam consumption, electrical power, thermal load.

\section{REFERENCES}

1. Kehlhofer R., Hannemann F., Stirnimann F., Rukes B. Combined Cycle Gas and Steam Turbine Power Plants. Tulsa, Oklahoma, USA, PennWell Corporation, 2009. 434 p.

2. Burmistrov N.A., Osipov R.A. Perspektivnye napravleniya perevooruzheniya deystvuyushchikh TES (teplovykh elektrostantsy) na baze gazovykh nadstroek [Promising directions of re-equipment of operating thermal power plants based on gas turbine toppings]. Elektroenergetika glazami molodezhi. Materialy VIII Mezhdunarodnoy nauchno-tekhnicheskoy konferentsii [Youth visioning for Electric Power Engineering. Proceedings of the eighth International science and technology conference]. Samara, 2017, pp. 112-113

3. Scherer V., Scherer D. The Gas Turbine GT26 in Combined Cycle application: conversion of a Coal Power Plant into a modem combined cycle firing natural gas and oil. International Gas Turbine Institute/American Society of Mechanical Engineers. Birmingham, 1996, $10 \mathrm{p}$.

4. Berezinec P.A. Gazoturbinnaya nadstroyka bloka $300 \mathrm{MVt}$ Kostromskoy GRES (gosudarstvennoy rayonnoy elektrostantsii) [Gas turbine topping of $300 \mathrm{MW}$ energy unit of Kostromskaya regional electric power plant]. Elektricheskie stantsii, 1999, no. 7, pp. 64-72.

5. Romashova O.Yu., Belyaev L.A., Tubolev A.A., Skrebatun E.A. Economic efficiency of a gas-turbine topping for steam reheating at heating turbo-installations. MATEC (Materials science, Engineering and Chemistry) Web of Conferences, 2017, vol. 91, $5 \mathrm{p}$.
6. Zaugolnikova Ya.A., Romashova O.Yu., Skrebatun E.A. Ekonomichnost ispolzovaniya gazoturbinnoy nadstroyki na energoblokakh teplovykh elektrostantsy [Economy of gas turbine topping use at thermal power plant energy units]. Intellektualnye energosistemy. Trudy III Mezhdunarodnogo molodezhnogo foruma [Smart Grids. Proc. of the third International youth forum]. Tomsk, 2015. Vol. 2, pp. 107-110.

7. Romashova O.Yu., Minor A.A., Martyshev V.N., Tubolev A.A. Efficiency of steam reheating in heat recovery steam generator at thermal power plant with gas turbine topping. MATEC (Materials science, Engineering and Chemistry) Web of Conferences, 2017, vol. $110,8 \mathrm{p}$.

8. Mohan G., Dahal S., Kumar U., Martin A., Kayal H. Development of natural gas fired combined cycle plant for tri-generation of power, cooling and clean water using waste heat recovery: Techno-economic analysis. Energies, 2014, no. 7, pp. 6358-6381.

9. Karaagac M.O., Kabul A., Yigit F. Performance Analysis of Natural Gas Combined Cycle Power Plant. Journal of Polytechnic - Politeknik Dergisi, 2019, vol. 22, pp. 319-325.

10. Romashova O.Yu., Tubolev A.A., Matveeva A.A., Zenkov A.V. Selection of optimal reheat temperature in heat recovery boiler at combined heat and power plant with gas turbine topping. MATEC (Materials science, Engineering and Chemistry) Web of Conferences, 2017, vol. 141, 4 p.

11. Goldberg A.A., Shibaev T.L., Paneke Agilera H.K., Stepanov M.Yu. Features of scheme solutions for steam turbine plants of ZAO «Ural Turbine Works», which are part of the CCPPs (combined cycle power plants). Teploenergetika, 2013, no. 8, pp. 27-31. In Rus. 
12. Khan M.N., Tlili I. New advancement of high performance for a combined cycle power plant: thermodynamic analysis. Case Studies in Thermal Engineering, 2018, no. 12, pp. 166-175.

13. Ibrahim T.K., Mohammed M.K., Awad O.I., Rahman M., Najafi G., Basrawi F., Alla A.N.A., Mamat R. The optimum performance of the combined cycle power plant: a comprehensive review. Renewable and Sustainable Energy Reviews, 2017, vol. 79, pp. 459474.

14. Javadi M.A., Ghomashi H. Thermodynamics analysis and optimization of Abadan combined cycle power plant. Indian Journal of Science and Technology, 2016, vol. 9, no. 7, 12 p.

15. Woudstra N., Woudstra T., Pirone A., Van der Stelt T. Thermodynamic evaluation of combined cycle plants. Energy Conversion and Management, 2010, vol. 51, no. 5, pp. 1099-1110.

16. Sobolev S.P. Parovaya turbina K-160-130 HTGZ (Kharkovskogo turbogeneratornogo zavoda) [Steam turbine K-160-130 HTGZ (Kharkov turbine generator plant)]. Moscow, Energiya Publ., 1980. $192 \mathrm{p}$.

17. Balabanovich V.K. Ohlazhdenie protochnoy chasti parovykh turbin na rezhimakh s minimalno-ventilyatsionnymi propuskami para [Cooling of steam turbines flow path on modes with minimum ventilation consumptions of steam]. Izvestiya vuzov. Energetika, 1981, no. 11, pp. 105-107.

18. Provedenie ekspluatatsionnykh ispytaniy turbiny T-178/210-130 st. № $12 \mathrm{~s}$ tselyu opredeleniya fakticheskoy ekonomichnosti $i$

Information about the authors

Alexander A. Minor, postgraduate student, National Research Tomsk Polytechnic University.

olga Yu. Romashova, Cand. Sc., associate professor, National Research Tomsk Polytechnic University. korrektirovki diagrammy rezhimov OAO «Surgutskaya GRES-1》 (gosudarstvennaya rayonnaya elektrostantsiya): tekhnicheskiy otchet [Performance tests of turbines T-178/210-130 st. no. 12 for defining actual efficiency and adjusting steam-consumption diagram of OAO «Surgut regional electric power plant-1»: technical report]. Ekaterinburg, 2008.97 p.

19. Ibrahim T.K., Rahman M.M., Abdalla A.N. Gas turbine configuration for improving the performance of combined cycle power plant. Procedia Engineering, 2011, vol. 15, pp. 4216-4223.

20. Canev S.V., Burov V.D., Zemtsov A.S., Osyka A.S. Gazoturbinnye energeticheskie ustanovki [Gas turbine power plants]. Moscow, Moscow Power Engineering Institute Publ. house, $2011.428 \mathrm{p}$.

21. Trukhniy A.D. Parogazovye ustanovki elektrostantsy [Steam-gas installations of power plants]. Moscow, Moscow Power Engineering Institute Publ. house, 2013. 648 p.

22. Olkhovskiy G.G., Chernetsky N.S., Berezinets P.A. Modernizatsiya energeticheskikh blokov putem ikh nadstroyki gazovymi turbinami [Redeveloping of energy units by their topping with gas turbines]. Elektricheskie stantsii, 1997, no. 4, pp. $9-18$.

Received: 12 September 2019. 